J. Korean Math. Soc. 50 (2013), No. 3, pp. 529-541

http://dx.doi.org/10.4134/JKMS.2013.50.3.529

\title{
AVOIDING PERMUTATIONS AND THE NARAYANA NUMBERS
}

\author{
Youngua Park and Seungkyung Park
}

\begin{abstract}
We study 132 avoiding permutations that also avoid $(2 r+$ 1) $(2 r+2) \cdots 12$ but contain $(2 r-1)(2 r) \cdots 12$ pattern. We find an identity between the number of these permutations and the Narayana number. We also present relations between 132 avoiding permutations and polygon dissections. Finally, a generalization of these permutations is obtained.
\end{abstract}

\section{Introduction}

Avoiding permutations of certain patterns have been studied over the years. Especially, the number of avoiding permutations of pattern 132 with $r$ descents is the Narayana number $N(n, k)$ (See [3]). We study 132-avoiding permutations that avoid another pattern but contain some other pattern.

Let $S_{n}$ be the set of permutations on $[n]:=\{1,2, \ldots, n\}$. For $\pi \in S_{n}$ and $\tau \in S_{k}$, we say that $\pi$ contains $\tau$-pattern if there exists a subsequence $1 \leq i_{1}<i_{2}<\cdots<i_{k} \leq n$ such that $\left(\pi_{i_{1}} \pi_{i_{2}} \cdots \pi_{i_{k}}\right)$ is in the same relative order as $\tau$. We say that $\pi$ is $\tau$-avoiding if such a subsequence does not exist. The set of all $\tau$-avoiding permutations in $S_{n}$ is denoted by $S_{n}(\tau)$. The set of all permutations containing $\tau$ in $S_{n}$ is denoted by $S_{n}(\bar{\tau})$. So $S_{n}\left(\sigma_{1}, \overline{\sigma_{2}}\right)$ is the set of all permutations avoiding $\sigma_{1}$ but containing $\sigma_{2}$ in $S_{n}$.

We consider the case of $\tau_{r}=(2 r-1)(2 r)(2 r-3)(2 r-2) \cdots 12$ pattern, and enumerate the number of permutations with $i$ ascents in $S_{n}\left(132, \tau_{r+1}, \overline{\tau_{r}}\right)$ for $r \geq 1$, and denote the set by $W_{n}^{i}(r)$. We generalize this set with the pattern $((r-1) d+1) \cdots(r d-1)(r d) \cdots(12 \cdots d)$ by $\tau_{r, d}$ and $W_{n}^{i}(r, d)$, the set of these permutations with $i$ ascents in $S_{n}\left(132, \tau_{r+1, d}, \overline{\tau_{r, d}}\right)$. We found that if $d=2$ in our formula, then the number is the same as in the following identity for $d=2$ obtained by Sulanke([5]):

$$
\prod_{i=0}^{d-1}\left(\begin{array}{c}
m+n+i \\
n
\end{array}\right)\left(\begin{array}{c}
n+i \\
n
\end{array}\right)^{-1}=\sum_{k \geq 0}\left(\begin{array}{c}
d n+m-k \\
d n
\end{array}\right) N(d, n, k),
$$

Received April 26, 2012; Revised September 14, 2012.

2010 Mathematics Subject Classification. 05A15.

Key words and phrases. avoiding permutation, Narayana number, dissection number. 
where $N(d, n, k)$ is the $d$-dimensional Narayana Numbers. He used Stanley's P-partitions theory and MacMahon's work. Callan [1] also found the same identity by counting Dyck paths according to the number of "plateaus". But for $d \geq 3$, our numbers are different from theirs.

In Section 2, we prove combinatorially that $N(n, i+1)=\sum_{r=1}^{i} w_{n}^{i}(r)$, where $\left|W_{n}^{i}(r)\right|=w_{n}^{i}(r)$ by Algorithms A, B, C, and D on avoiding permutations. Although we use the four algorithms, they give some applications on themselves. Moreover, we can generalize $W_{n}^{i}(r)$ further using those algorithms to get $W_{n}^{i}(r, d)$. If $d=2, W_{n}^{i}(r, 2)=W_{n}^{i}(r)$, which is the same as the results of Sulanke and Callan. We discuss this in Section 5.

Section 3 deals with the polygon dissection numbers derived by Algorithms $\mathrm{A} \sim \mathrm{D}$. In Section 4, we give the number of the avoiding permutations that have exactly one pattern of $\tau_{r}$.

\section{Avoiding permutations with the specific restrictions}

We call $i$ a descent (resp., ascent) of a permutation $\pi=\pi_{1} \cdots \pi_{n}$ if $\pi_{i}>\pi_{i+1}$ (resp., $\pi_{i}<\pi_{i+1}$ ) for $i=1,2, \ldots, n-1$ and count an extra descent at the end of each permutation. We denote by $\operatorname{des}(\pi)$ the number of descents in $\pi$.

An ascending run of a permutation is an increasing sequence of consecutive entries. Thus if $\pi$ has $k$ descents, then it has $k$ ascending runs. If $a_{i+1} \cdots a_{i+j}$ is an ascending run, then we say it is of length $j$. Specially, we call an ascending run with length more than 1 in 132 avoiding permutation a long ascending run. Note that any permutation can be decomposed into ascending runs, each of which is denoted by $I_{j}$. For example, if $\pi=5631247$, then $\pi=56|3| 1247$ has three ascending runs separated by bars, namely, $I_{1}=56, I_{2}=3$, and $I_{3}=1247$ of lengths 2,1 , and 4 , respectively. We represent by $I_{j} I_{k}$ the concatenation of two runs of $I_{j}$ and $I_{k}$.

Define $\tau_{r}:=(2 r-1)(2 r)(2 r-3)(2 r-2) \cdots 12$ for $r \geq 1$. Then $S_{n}\left(132, \tau_{r+1}, \overline{\tau_{r}}\right)$ is the set of all permutations that avoid the patterns 132 and $\tau_{r+1}$ but contain the pattern $\tau_{r}$. Thus any permutation in $S_{n}\left(132, \tau_{r+1}, \overline{\tau_{r}}\right)$ must have at least $r$ ascending runs of length at least 2 .

Let $W_{n}^{i}(r)$ be the set of permutations $\pi$ in $S_{n}\left(132, \tau_{r+1}, \overline{\tau_{r}}\right)$ satisfying $\operatorname{des}(\pi)$ $=n-i$. We write $w_{n}^{i}(r)$ for $\left|W_{n}^{i}(r)\right|$. Let $S_{n, r}(132)$ be the set of all permutations that avoid the pattern 132 and have $r$ descents. Krattenthaler [3] showed that the number of such permutations is the Narayana number $N(n, r)$.

If $\pi \in W_{n}^{i}(r)$, then $\pi=I_{1} \cdots I_{n-i}$, where each $I_{j}$ is an ascending run. Since $\pi$ contains the pattern $\tau_{r}$, there must be $r$ ascending runs, where the concatenation of any distinct pair of them contains a 3412 pattern. We call these the basic ascending runs. Notice that any ascending run of length 1 cannot be a basic ascending run, and that the first ascending run of length greater than 1 automatically becomes a basic ascending run.

The following theorem provides a closed formula for the number $w_{n}^{i}(r)$ using this fact. 
Theorem 2.1. For $i \geq r \geq 1, w_{n}^{i}(r)=N(i, r)\left(\begin{array}{l}n-r+i \\ n-r-i\end{array}\right)$, where $N(i, r)=$ $\frac{1}{i}\left(\begin{array}{c}i \\ r\end{array}\right)\left(\begin{array}{c}i \\ r-1\end{array}\right)$ is the Narayana number.

Proof. Algorithm A shows $\phi(\pi) \in S_{i, r}(132)$ and Algorithms B, C, and D show the map $\phi$ is $\left(\begin{array}{c}n-r+i \\ n-r-i\end{array}\right)$-to-one function. To define a map $\phi: W_{n}^{i}(r) \rightarrow S_{i, r}(132)$ for a permutation $\pi=a_{1} \cdots a_{n}=I_{1} \cdots I_{n-i} \in W_{n}^{i}(r)$ with the descent set $\left\{j_{1}, \ldots, j_{n-i}\right\}$, we apply the following algorithm to $\pi$ and the output $\phi(\pi)$ becomes an 132-avoiding permutation with $r$ descents.

If $I_{j}$ is a long ascending run and $I_{k}$ the next long ascending run, and if $I_{j} I_{k}$ does not contain a 3412 pattern, then we choose the first entry of $I_{k}$ as $d_{j}$ for $j=1,2, \ldots, n-i-1$. Otherwise, choose the last element from each of the long ascending runs. Let $\left\{d_{1}, d_{2}, \ldots, d_{k}, \ldots, d_{n-i}\right\}$ be the chosen entries. Notice that $d_{n-i}=a_{n}$. We now delete $d_{k}$ 's according to the following algorithm:

\section{Algorithm A}

STEP 1. Delete $d_{n-i}=a_{n}$.

STEP 2. For each $k=1,2, \ldots, n-i-1$,

(a) Subtract 1 from each entry before $d_{k}$, and subtract 1 from each entry greater than $d_{k}$ after $d_{k}$.

(b) If there exist $a_{s}$ before $d_{k}$ and $a_{t}$ after $d_{k}$ with $a_{s}=a_{t}$, then replace $a_{t}$ by the number $d_{k}-1$. Then remove $d_{k}$ from the ascending run.

Now we show that $\phi(\pi) \in S_{i, r}(132)$ and that the map is a $\left(\begin{array}{l}n-r+i \\ n-r-i\end{array}\right)$-to-one function.

Since we remove $n-i$ entries from $\pi$, clearly, $\phi(\pi)$ is a permutation on $i$ letters. Moreover, for each $k=1,2, \ldots, n-i-1$, the permutation after Step 2 is still 132-avoiding. Notice that after the one run of Step 2, every entry is distinct. If (b) of Step 2 does not occur, then clearly 132-avoiding is preserved. For the case of (b), every number appearing between $d_{k}-2$ and $d_{k}-1$ is less than $d_{k}-2$, which means that it preserves 132-avoiding after Step (b).

Notice also that all length 1 ascending runs in $\pi$ are removed by the algorithm. So ignore those runs and only consider the long ascending runs. Recall that the first long ascending run is the first basic ascending run. If the next ascending run is a basic ascending run, then removing the last entry in the first one does not change the descent. But if it is a nonbasic ascending run, which means that there is no 3412-pattern in the concatenation of the two, then removing the first element by Step 2 deletes the descent. So the next ascending run is merged to the first one, which becomes a basic ascending run with a bigger size. As we proceed with the algorithm, the number of basic ascending runs does not change.

Thus when the algorithm is completed, we obtain a permutation on $i$ letters with $r$ descents. In other words, $\phi(\pi) \in S_{i, r}(132)$. 
Secondly, we show that the map is a $\left(\begin{array}{l}n-r+i \\ n-r-i\end{array}\right)$-to-one function. Let $\phi(\pi)=$ $\sigma=b_{1} \cdots b_{i} \in S_{i, r}(132)$ and the descent set of $\sigma$ be $\operatorname{Des}(\sigma)=\left\{j_{1}, \ldots, j_{r}\right\}, j_{r}=$ $i$.

We will insert $r$ entries into $\sigma$ to obtain $\sigma^{*}=c_{1} \cdots c_{i+r} \in S_{i+r, r}(132)$, where $\operatorname{Des}\left(\sigma^{*}\right)=\left\{j_{1}+1, \ldots, j_{r}+r\right\}$. Let $\sigma=I_{1} \cdots I_{r}$, where $I_{k}$ 's are ascending runs and let $a_{k, l_{k}}$ be the last entry of $I_{k}$.

\section{Algorithm B}

STEP 1. For each $k=r-1, r-2, \ldots, 1$,

(a) Insert the number $a_{k, l_{k}}+1$ into $I_{k}$ as its last entry.

(b) Add 1 to each entry in $I_{j}$ including $a_{k, l_{k}}+1$ for $1 \leq j \leq k$, and add 1 to each entry in $I_{j}, j>k$, that is greater than or equal to $a_{k, l_{k}}+2$. We denote by $*$ the output of this step.

(c) If an entry $a_{s, i_{s}}^{*}$ in $I_{s}^{*}$ is the same as an entry $a_{t, i_{t}}^{*}$ in $I_{t}^{*}$ with $k \leq s<t$, then replace $a_{t, i_{t}}^{*}$ by $a_{s, 1_{s}}^{*}-1$, where $a_{s, 1_{s}}^{*}$ is the first entry of $I_{s}^{*}$. Repeat this process until all entries in $I_{j}^{*}, j \geq k$, become distinct.

STEP 2. Attach the unused number from $[i+r]=\{1,2, \ldots, i+r\}$ to the end of the last ascending run $I_{r}$, which becomes $I_{r}^{*}=c_{i+r}$.

The output $\sigma^{*}=c_{1} \cdots c_{i+r}=I_{1}^{*} \cdots I_{r}^{*}$ is a permutation on $i+r$ letters that avoids 132-pattern. Before Step 2, since every number between 1 and the largest element $a_{r, l_{r}}$ of $I_{r}^{*}$ is used from the construction, the unused number must be greater than $a_{r, l_{r}}$. Thus attaching the unused one to the end of $I_{r}^{*}$ does not produce a descent. Therefore, the number of descents of $\sigma^{*}$ is still $r$.

By (a), (b) of Step1 and Step 2, each $\left|I_{j}^{*}\right| \geq 2,1 \leq j \leq r$. By (c) of Step 1, each $I_{j}^{*}, 1 \leq j \leq r$, has at least two elements less than the first element of $I_{j-1}^{*}$. So it has $r$ basic ascending runs.

Therefore, $\sigma^{*}$ has $\tau_{r}$ pattern and $\sigma^{*}$ avoids $\tau_{r+1}$.

Now we insert $m(0 \leq m \leq n-i-r)$ numbers into the basic ascending runs of $\sigma^{*}=I_{1}^{*} \cdots I_{r}^{*}$ from right to left, creating $m$ descents, to obtain $\sigma^{* *}$ in Algorithm C.

\section{Algorithm C}

For each $m$ of $0,1, \ldots, n-i-r$, find all possible positions that are spaces between $a_{k, 2_{k}}$ and $a_{k, l_{k}}$ from each $I_{k}^{*}$ with repetition allowed for $k=1,2, \ldots, r-$ 1. For $k=r$, include one more space, the last space. Suppose that we select $t_{k}$ positions for each $k=r, r-1, \ldots, 1$ and $\sum_{k} t_{k}=m$ with $t_{k} \geq 0$. We insert $t_{k}$ numbers into the selected positions $I_{k}^{*}$.

For each $m=0,1, \ldots, n-i-r$ and for $k=r, r-1, \ldots, 1$,

STEP 1. For the chosen $t_{k}$ positions from the possible spaces from $I_{k}^{*}$, add the number $t_{k}$ to each entry in $\sigma^{*}$ that is greater than or equal to $a_{k, 1_{k}}$.

STEP 2. Insert $t_{k}$ numbers of $a_{k, 1_{k}}+\left(t_{k}-1\right), \ldots, a_{k, 1_{k}}+1, a_{k, 1_{k}}$ into the positions selected from right to left, keeping this decreasing order. 
Thus there are $\left(\left(\begin{array}{c}i-r+1 \\ m\end{array}\right)\right)=\left(\begin{array}{c}i-r+m \\ m\end{array}\right)$ ways to get $\sigma^{* *}$.

Finally, we construct $\sigma^{* * *}$ from $\sigma^{* *}$ to complete the proof. We insert $n-$ $i-r-m$ numbers in the fronts of $I_{k}^{* *}$ 's with repetition allowed from right to left in such a way that every time we place a number a new descent must be created.

Among $r+m$ ascending runs in $\sigma^{* *}$, there are $r$ basic ascending runs and we denote them by $I_{k}^{* *}=a_{k, 1_{k}} a_{k, 2_{k}} \cdots a_{k, l_{k}}$ for $1 \leq k \leq r$. Define $l_{k}$ as $l_{k}=$ $a_{k-1,1_{k-1}}-a_{k, 1_{k}}-t_{k-1}$ for $k=2,3, \ldots, r$, and $l_{1}=(i+r+m+1)-a_{1,1_{1}}$. Suppose that $u_{k}$ numbers $h_{1}, \ldots, h_{u_{k}}$ are placed in front of the $k$ th basic ascending run $I_{k}^{* *}$ and that $\sum_{k} u_{k}=n-i-r-m$. Then these numbers should satisfy the conditions $a_{k, 2_{k}}+u_{k}-j \leq h_{j} \leq a_{k, 2_{k}}+u_{k}-j+l_{k}-1$ and $h_{1}>h_{2}>\cdots>h_{u_{k}}$.

Suppose that we insert $u_{k}$ numbers in front of $I_{k}^{* *}$ for each $k=1,2, \ldots, r$ and let $\sum_{k=1}^{r} u_{k}=n-i-r-m$ with $u_{k} \geq 0$.

For each of $k=r, r-1, \ldots, 1$,

\section{Algorithm D}

STEP 1. Find $l_{k}$ for all $k=r, r-1, \ldots, 1$.

$\mathrm{STEP} 2$. For $k=r, r-1, \ldots, 1$,

(a) For $j=u_{k}, u_{k}-1, \ldots, 1$, find the range for $h_{j}$.

(b) Find all possible sequences with $h_{1}>h_{2}>\cdots>h_{u_{k}-1}>h_{u_{k}}$, then place each of such sequences in front of $I_{k}$ in the order of $h_{u_{k}}, h_{u_{k}-1}, \ldots, h_{2}, h_{1}$ from right to left.

(c) Each time $h_{j}$ is inserted, add 1 to each entry of $\sigma^{* *}$ that is greater than or equal to $h_{j}$.

After completing Algorithms B through D, we obtain a permutation $\sigma^{* * *}$ on $n$ letters and by our construction, it is clear that $\sigma^{* * *} \in W_{n}^{i}(r)$. Since $\sum_{k} l_{k}=i+r$, the total number of ways of inserting $n-i-r-m$ numbers into $i+r$ positions with repetition allowed is $\left(\left(\begin{array}{c}i+r \\ n-i-r-m\end{array}\right)\right)=\left(\begin{array}{c}n-m-1 \\ i+r-1\end{array}\right)$.

By Vandermonde's, we get

$$
\sum_{m=0}^{n-i-r}\left(\begin{array}{c}
i-r+m \\
m
\end{array}\right)\left(\begin{array}{c}
n-m-1 \\
i+r-1
\end{array}\right)=\left(\begin{array}{l}
n-r+i \\
n-r-i
\end{array}\right) .
$$

Thus the map $\phi$ is a $\left(\begin{array}{c}n-r+i \\ n-r-i\end{array}\right)$-to-one function.

Therefore, $w_{n}^{i}(r)=N(i, r)\left(\begin{array}{c}n-r+i \\ n-r-i\end{array}\right)=\frac{1}{i}\left(\begin{array}{c}i \\ r\end{array}\right)\left(\begin{array}{c}i \\ r-1\end{array}\right)\left(\begin{array}{c}n-r+i \\ n-r-i\end{array}\right)$.

Example 2.2. Apply Algorithm A to the following permutations $\pi$ to obtain $\phi(\pi)$.

$$
\begin{aligned}
& \pi=5 \mathbf{6}|\mathbf{3}| 124 \mathbf{7} \rightarrow 5 \mathbf{6}|\mathbf{3}| 124 \rightarrow 4|\mathbf{3}| 124 \rightarrow 4|\mathbf{3}| 125 \rightarrow 3124=\phi(5631247) . \\
& \pi=5 \mathbf{6}|234| \mathbf{1 7} \rightarrow 5 \mathbf{6}|234| \mathbf{1} \rightarrow 4|234| \mathbf{1} \rightarrow 4|235| \mathbf{1} \rightarrow 3124=\phi(5623417) .
\end{aligned}
$$

Example 2.3. Clearly, $S_{2,2}(132)=\{21\}$. We apply Algorithm B through $\mathrm{D}$ to find all $\pi$ 's on $n=6$ letters whose image is $\phi(\pi)=\sigma=21$. Since $0 \leq m \leq n-i-r=6-2-2=2$, we consider each of these three cases: 
First, apply Algorithm B to $\sigma=2 \mid 1$ to obtain $\sigma^{*}: \sigma=2|1 \rightarrow 23| 1 \rightarrow \mathbf{3 4} \mid 1 \rightarrow$ $34 \mid 12=\sigma^{*}$.

Now, if $m=2$, apply Algorithm $\mathrm{C}$ to $\sigma^{*}$ to get $\sigma^{* *}: \sigma^{*}=34|12 \rightarrow \mathbf{5 6}| \mathbf{3 4} \rightarrow$ $56 \mid 3421=\sigma^{* *}$. We have found a permutation in $W_{6}^{2}(2)$ so we do not need to apply Algorithm D.

For $m=1$, by Algorithm C, $\sigma^{*}=34|12 \rightarrow \mathbf{4 5}| 23 \rightarrow 45 \mid 231=\sigma^{* *}$. By Algorithm D, to insert in front of the first basic ascending run 45, $5 \leq h_{1} \leq 6$ since $l_{1}=i+r+m+1-a_{1,1_{1}}=2+2+1+1-4=2$. Similarly, to insert in front of the second basic ascending run $23,3 \leq h_{1} \leq 4$ since $l_{2}=a_{1,1_{1}}-a_{2,1_{2}}-0=$ $4-2=2$. Thus we will have four outputs of Algorithm D.

$\sigma^{* *}=45|23| 1 \rightarrow \mathbf{5} 45|23| 1 \rightarrow 546 \mid 231=\sigma^{* * *}$, or $\sigma^{* *}=45|23| 1 \rightarrow \mathbf{6 4 5}|23| 1$ $=\sigma^{* * *}, \sigma^{* *}=45|23| 1 \rightarrow 453|23| 1 \rightarrow \mathbf{5 6 3}|24| 1=\sigma^{* * *}, \sigma^{* *}=45|23| 1 \rightarrow$ $454|23| 1 \rightarrow 564|23| 1=\sigma^{* * *}$.

Finally, for $m=0$, we have to insert $n-i-r-m=6-2-2-0=2$ numbers according to Algorithm D into two positions of $\sigma^{* *}=34 \mid 12$ in front of $I_{1}=34(\mathrm{a}), I_{2}=12(\mathrm{~b})$, or one of each(c).

First, we find $l_{k}$ 's: $l_{1}=2$ and $l_{2}=2$.

Case (a): $5 \leq h_{1} \leq 6$ and $4 \leq h_{2} \leq 5$.

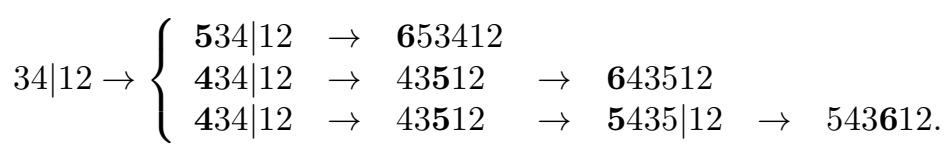

Case (b): $3 \leq h_{1} \leq 4$ and $2 \leq h_{2} \leq 3$.

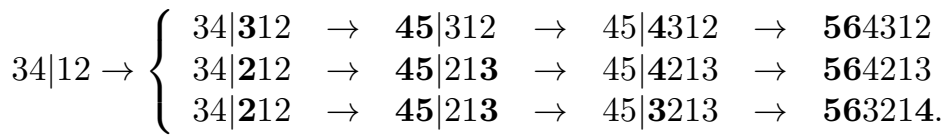

Case (C): for inserting $2 \leq h_{1} \leq 3$ in front of $I_{2}=12$, then for $5 \leq h_{1} \leq 6$ in front of the first run, one at a time.

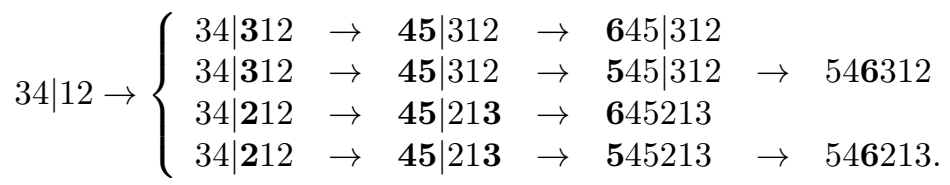

Thus we have

$$
\begin{gathered}
W_{6}^{2}(2)=\left\{\begin{array}{c}
563421,645231,546231,564231,563241,564312,564213,563214 \\
6
\end{array},\right. \\
653412,643512,543612,645312,546312,645213,546213\} .
\end{gathered}
$$

\section{Corollary 2.4.}

$$
\sum_{r=1}^{i} w_{n}^{i}(r)=\sum_{r=1}^{i} N(i, r)\left(\begin{array}{l}
n-r+i \\
n-r-i
\end{array}\right)=N(n, i+1) .
$$


TABLE $1 . w_{n}^{i}(r)$

\begin{tabular}{|c|c|c|c|c|c|c|c|c|c|c|c|c|c|c|c|}
\hline$n$ & 1 & 2 & & 3 & & & 4 & & & & 5 & & & & \\
\hline$r \backslash i$ & 0 & 0 & 1 & 0 & 1 & 2 & $\overline{0}$ & 1 & 2 & 3 & 0 & 1 & 2 & 3 & 4 \\
\hline 0 & 1 & 1 & 0 & 1 & 0 & 0 & 1 & 0 & 0 & 0 & 1 & 0 & 0 & 0 & 0 \\
\hline 1 & 0 & 0 & 1 & 0 & 3 & 1 & 0 & 6 & 5 & 1 & 0 & 10 & 15 & 7 & 1 \\
\hline 2 & 0 & 0 & 0 & 0 & 0 & 0 & 0 & 0 & 1 & 0 & 0 & 0 & 5 & 3 & 0 \\
\hline 3 & 0 & 0 & 0 & 0 & 0 & 0 & 0 & 0 & 0 & 0 & 0 & 0 & 0 & 0 & 0 \\
\hline 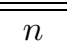 & \multicolumn{6}{|l|}{$\bar{~} 6$} & \multicolumn{7}{|l|}{7} & 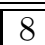 & \\
\hline$r \backslash i$ & 0 & 1 & 2 & 3 & 4 & 5 & 0 & 1 & 2 & 3 & 4 & 5 & 6 & \begin{tabular}{|l|l}
0 \\
\end{tabular} & 1 \\
\hline 0 & 1 & 0 & 0 & 0 & 0 & 0 & 1 & 0 & 0 & 0 & 0 & 0 & 0 & \begin{tabular}{|l|} 
\\
\end{tabular} & 0 \\
\hline 1 & 0 & 15 & 35 & 28 & 9 & 1 & 0 & 21 & 70 & 84 & 45 & 11 & 1 & 0 & 28 \\
\hline 2 & 0 & 0 & 15 & 21 & 6 & 0 & 0 & 0 & 35 & 84 & 54 & 10 & 0 & 0 & 0 \\
\hline 3 & 0 & 0 & 0 & 1 & 0 & 0 & 0 & 0 & 0 & 7 & 6 & 0 & 0 & 0 & 0 \\
\hline
\end{tabular}

Proof. Since $w_{n}^{i}(r)=N(i, r)\left(\begin{array}{c}n-r+i \\ n-r-i\end{array}\right)$,

$$
\sum_{r=1}^{i} w_{n}^{i}(r)=\sum_{r=1}^{i} N(i, r)\left(\begin{array}{c}
n-r+i \\
n-r-i
\end{array}\right) .
$$

Moreover, $\sum_{r=1}^{i} w_{n}^{i}(r)=S_{n, n-i}(132)$, where $S_{n, n-i}(132)$ is the set of 132 avoiding permutations on $n$ letters with $n-i$ descents. This is known to be $S_{n, n-i}(132)=N(n, n-i)=N(n, i+1)$ from [3].

Remark 2.5. From the definition of $w_{n}^{i}(r)$, we provide that

$$
\left|S_{n+2 r}\left(132, \tau_{r+1}, \overline{\tau_{r}}\right)\right|=\sum_{i=r}^{n+r} N(i, r)\left(\begin{array}{c}
n+r+i \\
n+r-i
\end{array}\right)
$$

for $r \geq 1, n \geq 0$. In particular, we take the sequence of $\left|S_{n+2}(132,3412, \overline{12})\right|=$ $F_{2(n+1)}-1$ is $\{1,4,12,33,88,232, \ldots\}$ for $n \geq 0, r=1$ because the sequence of $\left|S_{n+2}(132,3412)\right|=\left|S_{n+2}(132,3412, \overline{12}) \cup\{n(n-1) \cdots 21\}\right|=F_{2(n+1)}$ is $\{1,2,5,13,34,89, \ldots\}$ (see [6], [4]), where $n \geq 0$ and $F_{n}$ is the Fibonacci number.

\section{An application of the algorithms}

We denote the set of permutations with $j$ long ascending runs in $S_{n}(132)$ by $P(n, j)$. We write $p_{n}(j)$ for $|P(n, j)|$. Let $n=2 j+m(j, m \geq 0)$. A permutation with $j$ long ascending runs in 132 avoiding permutation has $s(0 \leq s \leq j-1)$ ascending runs and $j-s$ basic ascending runs. Suppose that $\pi \in P(2 j+m, j)$ has $t(0 \leq t \leq m)$ ascending runs with length 1 . Then $\pi$ has $j+m-t$ ascents. To obtain the number of permutations with $j$ long ascending runs in 132 avoiding permutation, we use Algorithms A, B, C, and D. 
TABLE 2. $\left|S_{n+2 r}\left(132, \tau_{r+1}, \overline{\tau_{r}}\right)\right|$

\begin{tabular}{|c|c|c|c|c|c|}
\hline$n \backslash r$ & 1 & 2 & 3 & 4 & 5 \\
\hline 0 & 1 & 1 & 1 & 1 & 1 \\
1 & 4 & 8 & 13 & 19 & 26 \\
2 & 12 & 42 & 102 & 205 & 366 \\
3 & 33 & 183 & 624 & 1650 & 3716 \\
4 & 88 & 717 & 3275 & 11020 & 30520 \\
5 & 232 & 2622 & 15473 & 64553 & 215481 \\
\hline
\end{tabular}

Proposition 3.1. Define $p_{m}(0)=1$ for $j=0$. Then for $j \geq 1$,

$$
p_{2 j+m}(j)=\sum_{t=0}^{m}\left[\sum_{s=0}^{j-1} N(j+m-t, j-s)\left(\begin{array}{c}
m-t+s \\
s
\end{array}\right)\right]\left(\begin{array}{c}
2 j+m \\
t
\end{array}\right),
$$

where $N(i, r)=\frac{1}{i}\left(\begin{array}{c}i \\ r\end{array}\right)\left(\begin{array}{c}i \\ r-1\end{array}\right)$ is the Narayana number and $i \geq r \geq 1$.

Proof. Every long ascending run is merged to a basic ascending run by Algorithm A. So $\pi \in P(2 j+m, j)$ becomes $\phi(\pi) \in S_{j+m-t, j-s}(132)$, where $j$ is the number of long ascending runs. By Algorithm $\mathrm{B}, j-s$ basic ascending runs are formed. So we obtain a $\sigma^{*} \in S_{2 j+m-t-s, j-s}(132)$. By making $s$ long ascending runs but not basic ascending run, we get $j$ long ascending runs in Algorithm C, where repetition is not allowed and the last space is excluded. Thus there are $\left(\begin{array}{c}j+m-t-(j-s) \\ s\end{array}\right)=\left(\begin{array}{c}m-t+s \\ s\end{array}\right)$ choices. Finally, by adding $t$ ascending runs with length 1 at the front of each ascending run and the last space in Algorithm D, where $l_{1}=2 j+m-t+1-a_{1,1_{1}}, l_{j+1}=a_{j, 1_{j}}, l_{k}=$ $a_{k-1,1_{k-1}}-a_{k, 1_{k}},(2 \leq k \leq j)$ and $\sum_{k=1}^{j+1} u_{k}=t, \sum_{k=1}^{j+1} l_{k}=2 j+m-t+1$. Also, $h_{q}=a_{k-1,1_{k-1}}+u_{k}-q$ at the last space and the front of ascending run, not basic ascending run. At the front of basic ascending run, $a_{k, 2_{k}}+u_{k}-q \leq h_{q} \leq$ $a_{k, 2_{k}}+u_{k}-q+\left(l_{k}-1\right)$. There are $\left(\left(\begin{array}{c}2 j+m-t+1 \\ t\end{array}\right)\right)=\left(\begin{array}{c}2 j+m \\ t\end{array}\right)$ choices. Therefore, we have $\sum_{t=0}^{m}\left[\sum_{s=0}^{j-1} N(j+m-t, j-s)\left(\begin{array}{c}m-t+s \\ s\end{array}\right)\right]\left(\begin{array}{c}2 j+m \\ t\end{array}\right)$.

Now we consider the inner summation $\left[\sum_{s=0}^{j-1} N(j+m-t, j-s)\left(\begin{array}{c}m-t+s \\ s\end{array}\right)\right]$. This counts the permutations with $j$ long ascending runs and not with ascending runs of length 1 in $S_{n}(132)$. Dyck $n$ path, $D_{n}$, is the set of paths on the square lattice with steps $(1,1)$ and $(1,-1)$ from $(0,0)$ to $(2 n, 0)$ that never falls below the $x$-axis. We denote steps $(1,1)$ and $(1,-1)$ by $U$ (upstep) and $D$ (downstep), respectively. We call a two consecutive step $U D$ a peak. By the bijection between $S_{n}(132)$ and Dyck $n$ paths $D_{n}$ in [3], we see that the above inner summation is equal to the number of paths with $j U D D$ s and $j$ peak $U D \mathrm{~s}$ in $D_{2 j+m-t}$. Callan [2] presented that dissections of a regular $(n+2)$ gon with $n-1-k$ noncrossing diagonals correspond to $k$-marked Dyck $n$ paths in one-to-one fashion. We insert $U D$ to each peak at $m-t$ marked Dyck 
$j+m-t$ path. Then we insert $U D$ to unmarked interior vertex after deleting the marked interior vertex, where we use interior vertices of down steps instead of up steps. So we expect that a Dyck $2 j+m-t$ path with $j U D D$ s and $j$ peak $U D$ s correspond to an $m-t$ marked Dyck path. Thus we can represent dissection numbers in terms of Narayana number.

\section{Corollary 3.2.}

$$
d_{j-1}(j+m-t+2)=\sum_{s=0}^{j-1} N(j+m-t, j-s)\left(\begin{array}{c}
m-t+s \\
s
\end{array}\right),
$$

where $j-1$ is the number of noncrossing diagonals in regular $(j+m-t+2)$ gon.

Therefore, Proposition 3.1 becomes:

Corollary 3.3. For $j \geq 1$,

$$
p_{2 j+m}(j)=\sum_{t=0}^{m} d_{j-1}(j+m-t+2)\left(\begin{array}{c}
2 j+m \\
t
\end{array}\right) .
$$

In fact, since the strings of $U U D$ and $U D D$ are equidistributed, $p_{n}(j)$ in $S_{n}(132)$ is equal to the number of Dyck $n$ paths with $j$ long ascents (A091156 $[4])$.

\section{Permutations with $\tau_{r}$ pattern exactly once in $S_{n+2 r-1}\left(132, \tau_{r+1}\right)$}

We count the number of permutations that contain $\tau_{r}$ pattern exactly once in $S_{n+2 r-1}\left(132, \tau_{r+1}\right)(n \geq 1, r \geq 2)$ by Algorithms $\mathrm{A} \sim \mathrm{D}$. We denote the set of permutations that contain $\tau_{r}$ pattern exactly once in $W_{n+2 r-1}^{i}(r)$ by $W_{n+2 r-1}^{1, i}(r)$. Let $E_{n}^{r}$ be the set of permutations that contains $\tau_{r}$ pattern exactly once in $S_{n+2 r-1}\left(132, \tau_{r+1}\right)$. We denote $\left|E_{n}^{r}\right|$ by $e_{n}^{r}(n \geq 1, r \geq 2)$. Then $e_{n}^{r}=$ $\sum_{i=r}^{n+r-1}\left|W_{n+2 r-1}^{1, i}(r)\right|$.

Proposition 4.1. Let $e_{1}^{r}=1, w_{i-t}^{0}(1)=1$ and $w_{i-t}^{0}(t)=0(t \geq 2)$. Then

$$
\begin{aligned}
e_{n}^{r} & =\sum_{i=r}^{n+r-1}\left(\sum_{t=1}^{r-1} w_{i-t}^{i-r}(t)\right)\left(\begin{array}{c}
n-1+i \\
n+r-1-i
\end{array}\right) \\
& =\sum_{i=r}^{n+r-1}\left(\sum_{t=1}^{r-1} N(i-r, t)\left(\begin{array}{c}
2 i-2 t-r \\
2 i-2 r
\end{array}\right)\right)\left(\begin{array}{c}
n-1+i \\
n+r-1-i
\end{array}\right) .
\end{aligned}
$$

Proof. Since each $W_{n}^{i}(r)$ is disjoint and $W_{n}^{i}(r)=\emptyset$ if $i<r$ or $n-i<r$, we know that each $W_{n+2 r-1}^{1, i}(r)$ are disjoint and $E_{n}^{r}=\dot{\cup}_{i=r}^{n+r-1} W_{n+2 r-1}^{1, i}(r)$. Since $\pi \in W_{n+2 r-1}^{1, i}(r)$ has $\tau_{r}$ pattern exactly once, the length of the first basic ascending run is 2 and other $r-1$ basic ascending runs have lengths greater than or equal to 2, where only the first two entries of each basic ascending run are less than the first entry of the preceding ascending run. Also, non-basic ascending runs of lengths greater than or equal to 2 must occur after the second basic 
ascending run. Otherwise, $\pi$ would have $\tau_{r}$ pattern more than once. To obtain $\left|W_{n+2 r-1}^{1, i}(r)\right|$, we use Algorithms $\mathrm{A} \sim \mathrm{D}$. All elements except the first element in each ascending run with length greater than or equal to 2 in $\phi(\pi)$ are larger than the elements before the ascending run. Each ascending run with length more than or equal to 3 in $\phi(\pi)$ has at least two elements less than the second elements after the ascending run. We consider each ascending run with length greater than or equal to 3 except the first element. Let $\phi(\pi)$ have $t$ ascending runs with length more than or equal to 3 . If we reverse the order of ascending runs, we regard $\phi(\pi)$ as a permutation in $W_{i-t}^{i-r}(t)$. So the number of $\phi(\pi)$ is $\left|W_{i-t}^{i-r}(t)\right|(1 \leq t \leq r-1)$ by the aforementioned reasons. There are $\left(\left(\begin{array}{c}i-r+1 \\ m\end{array}\right)\right)=$ $\left(\begin{array}{c}i-r+m \\ m\end{array}\right)$ choices for $\sigma^{* *}$. For $\sigma^{* * *}$, we change $a_{k, 2_{k}}$ of $I_{k}^{* *}$ in the range of $h_{j}$ into $a_{k, 2_{k}}+1$ and change $l_{k}-1$ into $l_{k}-2$ for exactly one $\tau_{r}$ pattern. So $a_{k, 2_{k}}+1+u_{t}-j \leq h_{j} \leq a_{k, 2_{k}}+1+u_{t}-j+l_{k}-2,(1 \leq j \leq t)$. Also, $\sum_{k=1}^{r}\left(l_{k}-1\right)=$ $i$. Then we get $\left(\left(\begin{array}{c}n+2 r-1-i-r-m \\ n+1\end{array}\right)\right)=\left(\begin{array}{c}n+r-2-m \\ n+r-1-i-m\end{array}\right)=\left(\begin{array}{c}n+r-2-m \\ i-1\end{array}\right)$ choices for $\sigma^{* * *}$. By Vandermonde's, we get $\sum_{m=0}^{n+r-1-i}\left(\begin{array}{c}i-r+m \\ m\end{array}\right)\left(\begin{array}{c}n+r-2-m \\ i-1\end{array}\right)=\left(\begin{array}{c}n-1+i \\ n+r-1-i\end{array}\right)$. Therefore, for $n \geq 2, r \geq 2$,

$$
\begin{aligned}
\left|E_{n}^{r}\right|=e_{n}^{r} & =\sum_{i=r}^{n+r-1}\left|W_{n+2 r-1}^{1, i}(r)\right|=\sum_{i=r}^{n+r-1}\left(\sum_{t=1}^{r-1} w_{i-t}^{i-r}(t)\right)\left(\begin{array}{c}
n+i-1 \\
n+r-1-i
\end{array}\right) \\
& =\sum_{i=r}^{n+r-1}\left(\sum_{t=1}^{r-1} N(i-r, t)\left(\begin{array}{c}
2 i-2 t-r \\
2 i-2 r
\end{array}\right)\right)\left(\begin{array}{c}
n-1+i \\
n+r-1-i
\end{array}\right) .
\end{aligned}
$$

In particular, if $r=2$,

$$
\begin{aligned}
e_{n}^{2} & =\sum_{i=2}^{n+1}\left(\begin{array}{c}
n-1+i \\
n+1-i
\end{array}\right)=F_{2 n}-1 \\
& =\left|S_{n+1}(132,3412, \overline{12})\right|=\sum_{i=1}^{n} N(i, 1)\left(\begin{array}{c}
n+i \\
n-i
\end{array}\right) .
\end{aligned}
$$

\section{A generalization}

We generalize Theorem 2.1 to the case of any length $d \geq 3$. Let us denote the pattern $((r-1) d+1) \cdots(r d-1)(r d) \cdots(12 \cdots d)$ by $\tau_{r, d}$ and write $W_{n}^{i}(r, d)$ for the set of these permutations with $i$ ascents in $S_{n}\left(132, \tau_{r+1, d}, \overline{\tau_{r, d}}\right)$, and $\left|W_{n}^{i}(r, d)\right|=w_{n}^{i}(r, d)$. We use Algorithms A, B, C, and D again. Since $\pi \in$ $W_{n}^{i}(r, d)$, there should be $r$ ascending runs, where the concatenation of any distinct pair of them contains a $(d+1 \cdots 2 d)(12 \cdots d)$ pattern. We call them the $d$-basic ascending runs. Notice that any ascending run of length $1, \ldots, d-1$ cannot be a basic ascending run, and that the first ascending run of length greater than $d-1$ automatically becomes a basic ascending run. 
Proposition 5.1. For $d \geq 3$,

$$
w_{n}^{i}(r, d)=\sum_{j=0}^{i-(d-1) r} w_{i}^{i-r-j}(r, d-1)\left(\begin{array}{l}
n-j-r+i \\
n-j-r-i
\end{array}\right) .
$$

Proof. By Algorithm A, $\pi \in W_{n}^{i}(r, d)$ becomes $\phi(\pi) \in W_{i}^{i-r-j}(r, d-1)$, where $j$ is the number of ascending runs with length greater than or equal 2 which are not $d$-basic ascending runs. So $0 \leq j \leq \operatorname{Min}(i-(d-1) r, n-i-r)$. By Algorithm B, we obtain a $\sigma^{*} \in S_{i+r+j, r+j}\left(132, \tau_{r+1, d}, \overline{\tau_{r, d}}\right)$ from $\sigma \in W_{i}^{i-r-j}(r, d-1)$. We use Algorithms C and D to increase $n-i-r-j$ descents. By Vandermonde's, we get

$$
\sum_{m=0}^{n-i-r-j}\left(\begin{array}{c}
i-r-j+m \\
m
\end{array}\right)\left(\begin{array}{c}
n-m-1 \\
i+r+j-1
\end{array}\right)=\left(\begin{array}{c}
n-r-j+i \\
n-r-j-i
\end{array}\right) .
$$

Therefore,

$$
w_{n}^{i}(r, d)=\sum_{j=0}^{i-(d-1) r} w_{i}^{i-r-j}(r, d-1)\left(\begin{array}{l}
n-j-r+i \\
n-j-r-i
\end{array}\right) .
$$

We know that for fixed $n, i$, and $d$, the sum of $w_{n}^{i}(r, d)$ for all $r \geq 0$ is also a

\begin{tabular}{|c|c|c|c|c|c|c|c|c|c|c|c|c|c|c|c|}
\hline$n$ & 1 & \multicolumn{2}{|l|}{2} & \multicolumn{3}{|l|}{3} & \multicolumn{4}{|l|}{4} & \multicolumn{5}{|l|}{5} \\
\hline$r \backslash i$ & 0 & 0 & 1 & 0 & 1 & 2 & 0 & 1 & 2 & 3 & 0 & 1 & 2 & 3 & 4 \\
\hline 0 & 1 & 1 & 1 & 1 & 3 & 0 & 1 & 6 & 1 & 0 & 1 & 10 & 5 & 0 & 0 \\
\hline 1 & 0 & 0 & 0 & 0 & 0 & 1 & 0 & 0 & 5 & 1 & 0 & 0 & 15 & 10 & 1 \\
\hline $\bar{~} \bar{n}$ & $\bar{~} 6$ & & & & & & 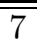 & & & & & & & 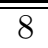 & \\
\hline$r \backslash i$ & 0 & 1 & 2 & 3 & 4 & 5 & 0 & 1 & 2 & 3 & 4 & 5 & 6 & 0 & 1 \\
\hline 0 & 1 & 15 & 15 & 1 & 0 & 0 & 1 & 21 & 35 & 7 & 0 & 0 & 0 & 1 & 28 \\
\hline 1 & 0 & 0 & 35 & 49 & 14 & 1 & 0 & 0 & 70 & 168 & 96 & 18 & 1 & 0 & 0 \\
\hline 2 & 0 & 0 & 0 & 0 & 1 & 0 & 0 & 0 & 0 & 0 & 9 & 3 & 0 & 0 & 0 \\
\hline
\end{tabular}
Narayana number.

TABLE 3. $w_{n}^{i}(r, 3)$

For the case when $d=3$ and $r=0$,

$$
\left|S_{n+3 r}\left(132, \tau_{r+1,3}, \overline{\tau_{r, 3}}\right)\right|=\left|S_{n}(132,123)\right|=2^{n} \text { (A011782 on OEIS). }
$$

For the case when $d=4$ and $r=0$,

$$
\left|S_{n+3 r}\left(132, \tau_{r+1,4}, \overline{\tau_{r, 4}}\right)\right|=\left|S_{n}(132,1234)\right|=F_{2 n} \text { (A001519 on OEIS), }
$$

where $F_{n}$ is the Fibonacci number.

These two cases present well-known numbers, but all other numbers of $r \geq 1$ and $d \geq 3$ appear to be new. 
TABLE $4 . w_{n}^{i}(r, 4)$

\begin{tabular}{|c|c|c|c|c|c|c|c|c|c|c|c|c|c|c|c|}
\hline$n$ & 1 & \multicolumn{2}{|l|}{2} & \multicolumn{3}{|l|}{3} & \multicolumn{4}{|l|}{4} & \multicolumn{5}{|l|}{5} \\
\hline$r \backslash i$ & 0 & 0 & 1 & 0 & 1 & 2 & 0 & 1 & 2 & 3 & 0 & 1 & 2 & 3 & 4 \\
\hline 0 & 1 & 1 & 1 & 1 & 3 & 1 & 1 & 6 & 6 & 0 & 1 & 10 & 20 & 3 & 0 \\
\hline 1 & 0 & 0 & 0 & 0 & 0 & 0 & 0 & 0 & 0 & 1 & 0 & 0 & 0 & 7 & 1 \\
\hline $\bar{n}$ & $\bar{~} 6$ & & & & & & $\overline{77}$ & & & & & & & $\overline{88}$ & \\
\hline$r \backslash i$ & 0 & 1 & 2 & 3 & 4 & 5 & 0 & 1 & 2 & 3 & 4 & 5 & 6 & 0 & 1 \\
\hline 0 & 1 & 15 & 50 & 22 & 1 & 0 & 1 & 21 & 105 & 91 & 15 & 0 & 0 & 1 & 28 \\
\hline 1 & 0 & 0 & 0 & 28 & 14 & 1 & 0 & 0 & 0 & 84 & 90 & 21 & 1 & 0 & 0 \\
\hline 2 & 0 & 0 & 0 & 0 & 0 & 0 & 0 & 0 & 0 & 0 & 0 & 0 & 0 & 0 & 0 \\
\hline
\end{tabular}

TABLE 5. $S_{n+3 r}\left(132, \tau_{r+1,3}, \overline{\tau_{r, 3}}\right), S_{n+4 r}\left(132, \tau_{r+1,4}, \overline{\tau_{r, 4}}\right)$

\begin{tabular}{|c|c|c|c||c|c|c|c|}
\hline \multicolumn{4}{|c||}{$d=3$} & \multicolumn{3}{c|}{$d=4$} \\
\hline$n \backslash r$ & 0 & 1 & 2 & $n \backslash r$ & 0 & 1 & 2 \\
\hline 0 & 1 & 1 & 1 & 0 & 1 & 1 & 1 \\
1 & 2 & 6 & 12 & 1 & 2 & 8 & 16 \\
2 & 4 & 26 & 89 & 2 & 5 & 43 & 151 \\
3 & 8 & 99 & 527 & 3 & 13 & 196 & 1100 \\
4 & 16 & 353 & 2736 & 4 & 34 & 819 & 6853 \\
5 & 32 & 1213 & 13036 & 5 & 89 & 3249 & 38455 \\
6 & 64 & 4078 & 58489 & 6 & 233 & 12464 & 200306 \\
\hline
\end{tabular}

By successively plugging in the initial condition $w_{n}^{i}(r, 2)=N(i, r)\left(\begin{array}{l}n-r+i \\ n-r-i\end{array}\right)$, which is Theorem 2.1, we obtain the following:

Corollary 5.2. Let $\alpha_{k}=j_{1}+\cdots+j_{k}, j_{l} \geq 0,1 \leq k \leq d-2, \alpha_{0}=0$.

$$
\begin{aligned}
w_{n}^{i}(r, d)= & \sum_{\alpha_{d-2} \leq i-(d-1) r}\left[N\left(i-(d-2) r-\alpha_{d-2}, r\right)\right. \\
& \left.\times \prod_{q=1}^{d-2}\left(\begin{array}{c}
2\left(i-q r-\alpha_{d-2}\right)+\alpha_{d-2}-\alpha_{q-1} \\
2\left(i-q r-\alpha_{q}\right)
\end{array}\right)\left(\begin{array}{c}
n-\alpha_{1}-r+i \\
n-\alpha_{1}-r-i
\end{array}\right)\right] .
\end{aligned}
$$

In particular, when $d=3$ we have:

\section{Corollary 5.3.}

$$
w_{n}^{i}(r, 3)=\sum_{j=0}^{i-2 r} N(i-r-j, r)\left(\begin{array}{c}
2 i-2 r-j \\
j
\end{array}\right)\left(\begin{array}{c}
n-j-r+i \\
n-j-r-i
\end{array}\right) .
$$

Acknowledgements. We would like to thank anonymous referee for his/her helpful comments. 


\section{References}

[1] D. Callan, Kreweras' Narayana Number Identity Has a Simple Dyck Path Interpretation, (2005), preprint.

[2] Polygon Dissections and marked Dyck Paths, (2005), preprint.

[3] C. Krattenthaler, Permutations with restricted patterns and Dyck paths, Adv. in Appl. Math. 27 (2001), no. 2-3, 510-530.

[4] N. J. A. Sloane, On-line Encyclopedia of Integer Sequences, http://www.research. att.com/ njas/sequences/index.html

[5] R. A. Sulanke, Generalizing Narayana and Schröder Numbers to Higher Dimensions, The Electronic Journal of Combinatorics 11 (2004), Art. R54.

[6] J. West, Generating trees and forbidden subsequences, Discrete Math. 157 (1996), no. $1-3,363-374$

YOUNGJA PARK

Department of Mathematics

YONSEI UNIVERSITY

SEOUl 120-749, KoreA

E-mail address: ojpark@yonsei.ac.kr

SEungkyung PARK

Department of Mathematics

Yonsei UNIVERSITY

SeOul 120-749, Korea

E-mail address: sparky@yonsei.ac.kr 